\title{
IDENTIFICAÇÃO DE PROBLEMAS RELACIONADOS A MEDICAMENTOS APRESENTADOS DURANTE O TRATAMENTO FARMACOTERAPEUTICO DAS GESTANTES COM TOXOPLASMOSE DA CIDADE DE CAMPOS DOS GOYTACAZES, RJ
}

\author{
Ana Carolina Miranda Ribeiro ${ }^{1}$, Jaíse Silva Ferreira ${ }^{2}$ \\ ${ }^{1}$ Aluna do Curso de Graduação em Farmácia da Faculdade de Medicina de Campos da FMC \\ ${ }^{2}$ Especialista em Manipulação magistral, Homeopatia e Atenção Farmacêutica. Orientadora e Professora do Curso de \\ Graduação em Farmácia da FMC
}

\section{RESUMO}

A toxoplasmose adquire enorme relevância quando ocorre durante a gestação pelo risco de transmissão vertical. Pode causar sequelas imediatas ou tardias, com manifestações neurológicas e coriorretinite, que podem se apresentar na segunda ou terceira décadas de vida. O objetivo do presente estudo foi Identificar os Problemas Relacionados aos Medicamentos (PRMs) apresentados pelas pacientes gestantes com toxoplasmose atendidas no Centro de Referência de toxoplasmose da cidade de Campos dos Goytacazes, RJ, O estudo foi do tipo observacional transversal com coleta de dados por meio de observação de prontuários e entrevista com 30 gestantes diagnosticadas com toxoplasmose que fazem tratamento farmacoterapêutico e acompanhamento no Ambulatório de Referência para Toxoplasmose em Campos dos Goytacazes, RJ. Observou-se a existência de PRM's nas gestantes participantes do estudo, 17 (56,6\%) apresentaram o PRM5, 10 (33,3\%) o PRM4 e apenas $1(3,33 \%)$ apresentou o PRM1.Conclusão:Nas gestantes com diagnóstico de toxoplasmose analisadas no presente estudo foram encontrados os PRM's do tipo 5, 4 e 1.0 acompanhamento farmacoterapêutico realizado por um farmacêutico possibilita uma aproximação real do paciente com relação ao seu próprio esquema terapêutico, facilitando a compreensão da importância da tomada correta dos medicamentos, com um esquema de administração orientado, minimizando as interações medicamentosas e consequentemente os problemas relacionados aos medicamentos.

Palavras-chave: Problemas Relacionados a Medicamentos. Gestante. Toxoplasmose.

\begin{abstract}
Toxoplasmosis acquires great relevance when it occurs during gestation due to the risk of vertical transmission. It may cause immediate or late sequelae, with neurologic manifestations and chorioretinitis, which may occur in the second or third decades of life. The objective of the present study was to identify the Problems Related to Medications (PRMs) presented by pregnant patients with toxoplasmosis treated at the Toxoplasmosis Reference Center of the city of Campos dos Goytacazes, RJ. The study was cross-sectional observational data collection Of observation of medical records and interview with 30 pregnant women diagnosed with toxoplasmosis who undergo pharmacotherapeutic treatment and follow up at the Reference Outpatient Clinic for Toxoplasmosis in Campos dos Goytacazes, RJ. PRMs were observed in the pregnant women participating in the study, 17 (56.6\%) presented PRM5, 10 (33.3\%) had PRM4 and only $1(3.33 \%)$ had PRM1. Conclusion: In pregnant women With the diagnosis of toxoplasmosis analyzed in the present study, PRMs of type 5, 4 and 1 were found. The pharmacotherapeutic follow-up performed by a pharmacist enables a real approximation of the patient in relation to his own therapeutic scheme, facilitating the understanding of the importance of the correct Drugs, with a targeted administration scheme, minimizing drug interactions and consequently drugrelated problems.
\end{abstract}

Keywords: Drug Related Problems. Pregnant. Toxoplasmosis. 


\section{INTRODUÇÃO}

O parasitaToxoplasma gondii (T. gondii) é um protozoário coccídeo que infecta um terço da população mundial, sendo uma doença de alta infecciosidade e baixa patogenicidade. O homem é geralmente infectado pelo consumo de carne crua ou mal cozida contaminada com cistos ou pela ingestão de água, alimentos ou solo contaminados com oocistos ou de T.gondii (CÂMARA; SILVA; CASTRO, 2014).

A toxoplasmose adquire enorme relevância quando ocorre durante a gestação pelo risco de transmissão vertical. Pode causar sequelas imediatas ou tardias, com manifestações neurológicas e coriorretinite, que podem se apresentar na segunda ou terceira décadas de vida. Por outro lado, em gestantes, a infecção é, na maioria dos casos, assintomática, havendo sintomas em apenas $10 \%$ dos casos. No início da gestação, a taxa de infecção fetal é pequena. No entanto, quando ocorre, as manifestações clínicas são graves, podendo resultar na morte do feto e no aborto espontâneo (CÂMARA; SILVA; CASTRO, 2014).

Estima-se que no Brasil o índice de toxoplasmose vem aumentando desde 2011, considerando que, em gestantes no país, no ano de 2011-2012 ocorreram variações entre 56,4 \% a 91,6 $\%$ (CÂMARA; SILVA; CASTRO, 2014). Esse índice pode ser comparado ao ano de 2005 que foi de $43,0 \%$ (NASCIMENTO et al.,2002).

O tratamento medicamentoso recomendado é a espiramicina, de acordo com maioria dos trabalhos e protocolos existentes e associada ao uso do esquema tríplice: pirimetamina, sulfadiazina e ácido folínico. Acredita-se que a falta de um protocolo resultou na prescrição da espiramicina sem associações podendo gerar Problemas Relacionados a Medicamentos (PRM's).

A utilização de medicamentos em gestantes só com confirmação laboratorial de toxoplasmose, a importância de um protocolo e um acompanhamento durante as consultas pode evitar possíveis efeitos adversos e uso de medicamentos desnecessários (MARGONATOL et al., 2008).

Uma droga ideal para o tratamento da toxoplasmose congênita deve apresentar propriedades parasiticidas contra os estágios do parasita, possuir uma penetração muito efetiva e, principalmente, não ser tóxica ao feto. No entanto, nenhuma droga utilizada durante o tratamento apresenta todas essas características (COSTA, 2009).

A toxoplasmose em gestantes é tratada com espiramicina $3 \mathrm{~g}$ /dia que é um macrolídeo ativo contra o Toxoplasma gondii, alcança elevada concentração na placenta e foi usada na Europa por longo tempo sem demostrar efeitos fetais. Se a toxoplasmose for identificada no feto e a gestação continuar, deverá ser iniciado $50 \mathrm{mg}$ de pirimetamina e $3 \mathrm{~g}$ de sulfadiazina por três semanas e acompanhada de $50 \mathrm{mg}$ de ácido folínico por dia (SÁ; JÚNIOR; NETTO, 1997).

O uso de medicamentos tem um papel importante na prevenção, manutenção e recuperação da saúde do paciente. No entanto, apesar desses benefícios a prescrição e a utilização incorreta de medicamentos constituem uma das principais causas de complicações à saúde, falhas na farmacoterapia ocasionando PRMs (AIZENSTEIN; TOMASSI, 2011). Durante o tratamento das gestantes com toxoplasmose podem ser apresentados alguns efeitos colaterais dos seguintes medicamentos (ANVISA,2016):

- Pirimetamina - possui efeitos significativos e prejudiciais à saúde como: arritmias cardíacas; náusea; anorexia; cólica; e diminuição dos glóbulos vermelhos; - Sulfadiazina - muito comum ocorrer dor de cabeça; perda de apetite; tontura; coceira; anemia; e febre;

Espiramicina - é um medicamento bem tolerado pelo organismo, por isso sua administração na dose correta não causa efeitos prejudiciais à saúde, já em doses acima da janela terapêutica pode ocorrer alguns efeitos como: náusea; vomito; diarreia; anorexia e boca seca.

Este estudo poderá contribuir para prevenção de PRMs apresentados pela população gestante portadora de doenças causadas pelo toxoplasma gondii, além de auxiliar na detecção de PRMs na farmacoterapia de toxoplasmose, fornecer informações dos PRMs de maior prevalência aos profissionais de saúde e identificar os PRM's apresentados pelas pacientes gestantes com toxoplasmose atendidas no Centro de Referência de toxoplasmose da cidade de Campos dos Goytacazes, RJ.

\section{MATERIAL E MÉTODO}

O trabalho compreende um estudo do tipo observacional transversal, com coleta de dados por meio de observação de prontuários e entrevista com 30 gestantes diagnosticadas com toxoplasmose que fazem tratamento farmacoterapêutico e acompanhamento no Ambulatório de Referência para Toxoplasmose em Campos dos Goytacazes, RJ. O procedimento para coleta de dados foi dividido em duas etapas, a primeira etapa foi a realização de entrevistas a partir de um protocolo (APENDICE A), nos dias de 
atendimento ambulatorial, durante os meses de março e abril de 2017 nos dias de terça-feira e quarta-feira, nos horários de 9:00hás 10:00h da manhã, no referido local e os dados obtidos registrados em protocolo de pesquisa.

No período que em ocorreu a coleta de dados para a presente pesquisa, o Ambulatório de Referência para Toxoplasmose, onde foi realizado o estudo, atendeu 30 gestantes com Toxoplasmose que participaram do estudo. As variáveis coletadas referentes à população investigada foram iniciais do nome, idade, período de gestação, início do tratamento e contato com animais de estimação, acompanhamento pré-natal, hábitos de prevenção da doença, medicamentos utilizados para tratamento de toxoplasmose, horário que utiliza os medicamentos, desconfortos durante o tratamento, desconfortos causados pelo(s) medicamento(s); PRMs identificados. Após a entrevista, as informações foram complementadas com a pesquisa no prontuário das pacientes sobre a prescrição e posologia dos medicamentos em uso.

Os PRM's foram analisados e identificados através de um protocolo elaborado com a classificação de cada um deles, de acordo com o prontuário e o questionário respondido pelas gestantes. Os PRM's foram classificados de acordo com a classificação do Segundo Consenso de Granada de 2002.

Os dados obtidos foram tabulados e apresentados em gráficos e tabelas elaboradas no Programa Excel. A análise realizada a partir do percentual da frequência de ocorrência dos PRMs identificados. Antes da realização da pesquisa, um projeto foi encaminhado para o Comitê de Ética em Pesquisa com Seres Humanos da Faculdade de Medicina de Campos e aprovado com o parecer número 62867016.2.0000.5244.

\section{RESULTADOS E DISCUSSÃO}

Foram entrevistadas 30 gestantes nos dias de atendimento para tratamento e acompanhamento para toxoplasmose, com faixas etárias de, 15-20 anos 9 (30\%), 20-25 anos 12 (40\%) e 25-30 anos $9(30 \%)$. Das gestantes, $30(100 \%)$ realizaram pré-natal, 11 (36\%) iniciaram o tratamento e acompanhamento no $1^{\circ}$ trimestre de gestação, $14(47 \%)$ no $2^{\circ}$ trimestre e 5 $(17 \%)$ no $3^{\circ}$ trimestre.

Sobre o período de gestação das pacientes 15 (50\%) estão no $3^{\circ}$ trimestre de sua gestação, $5(17 \%)$ no $1^{\circ}$ trimestre e $10(33 \%)$ no $2^{\circ}$ trimestre. Uma vez que a toxoplasmose geralmente é assintomática passando clinicamente despercebida as gestantes normalmente demoram a procurar um tratamento e chegar ao diagnóstico final.

No presente estudo a maioria das gestantes entrevistadas $22(73,3 \%)$ possuem contato direto com animais de estimação,15(50\%) delas possuem contato com gatos e $7(23,3 \%)$ com outros animais, tais como: cachorros e galinhas.

A toxoplasmose é uma doença de origem alimentar mesmo quando não diretamente relacionada a alimentação, entretanto a importância de verificar os fatores de risco é inquestionável para medidas de cuidados e orientações das pessoas. $\mathrm{Na}$ amostra pesquisada $30(100 \%)$ das gestantes afirmaram que lavavam as mãos após contato com animais e lavavam utensílios de cozinha, porém apenas $10(33,3 \%)$ das gestantes não se alimentavam de carnes cruas ou mal cozida e 11 (36\%) relataramoutros, como: contato com rios, bebe água não fervida e água de poço (Figura 1).

Confirmada a suspeita de Toxoplasmose congênita é instituído o tratamento medicamentoso, com o uso de Sulfadiazina; Pirimetamina; Ácido folínico; Espiramicina; e se necessário Prednisona. Nas gestantes pesquisadas, em alguns casos foram utilizados a Clindamicina, já que a Espiramicina ficou em falta na farmácia municipal durante alguns meses, e o tratamento não pode parar. Cinco gestantes fizeram o uso desse fármaco (Tabela 1).

O tratamento para toxoplasmose é de suma importância para que as sequelas não sejam agressivas, sem o uso de medicamentos a infecção pode levar ao, abortamento do feto, atraso mental, calcificações, deficiências visuais e microcefalia (MARTINS, 2002).

A Espiramicina é utilizada em gestantes na fase aguda da gestação, não prejudica o feto pois não atravessa a barreira placentária, já o Esquema Tríplice não deve ser administrado em gestantes com período inferior a 18 semanas de gestação, pois pode causar alterações embrionárias e a Clindamicina foi indicada na amostra estudada para algumas gestantes, considerando a necessidade de continuação do tratamento na falta da Espiramicina que é o medicamento ideal para a doença, foi observado que a Espiramicina causou $65 \%$ dos efeitos adversos nas gestantes, Esquema Tríplice $20 \%$ e a Clindamicina $15 \%$ (Figura 2).

É importante ressaltar, o relato de $19(63,3 \%)$ das gestantes sobre fazerem a ingestão de água de bica. Esse é um fator de risco importante relacionado a causa toxoplasmose. 


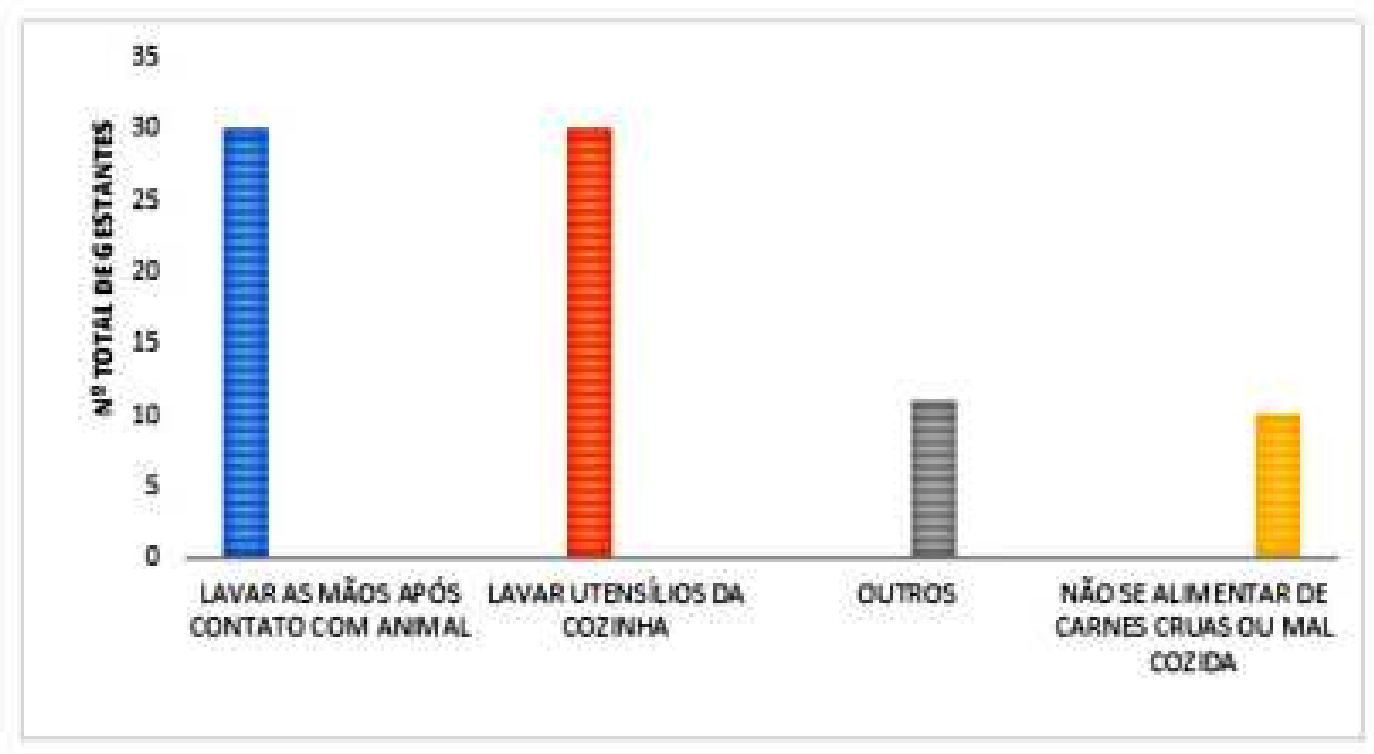

Fig 1- hábitos realizados pelas gestantes de prevençâo da doença.

Tabela 1- Medicamentos utilizados durante o tratamento farmacoterapêutico das gestantes com diagnóstico de toxoplasmose

\begin{tabular}{c|c|c}
\hline MEDICAMENTOS & $\begin{array}{c}\mathrm{N}^{0} \text { DE } \\
\text { GESTANTES }\end{array}$ & $\%$ \\
\hline Espiramicina & 20 & 66,6 \\
\hline $\begin{array}{c}\text { Esquema Triplice } \\
\begin{array}{c}\text { (Sulfadiazina+Pirimetamina+Acido } \\
\text { folinico) }\end{array}\end{array}$ & 10 & 33,3 \\
\hline Clindamicina & 5 & 17 \\
\hline Rifamicina & 4 & 13,3 \\
\hline Vitamina C & 2 & 6,6 \\
\hline
\end{tabular}

Os medicamentos utilizados durante o tratamento para toxoplasmose possuem efeitos significativos e são prejudiciais à saúde tais, como: náusea; cólica; diarréia; reações na pele; vômito, dor de cabeça e coceira (ANVISA,2016).

No presente estudo $20(66,6 \%)$ das gestantes apresentaram alguns desses efeitos colaterais durante o tratamento (Figura 3).

Segundo Consenso de Granada, 2002 os PRMs são classificados como (Figura.4):

PRM1. O paciente sofre um problema de saúde em conseqüência de não receber um medicamento de que necessita.
PRM2. O paciente sofre um problema de saúde em conseqüência de receber um medicamento de que não necessita.

PRM3. O paciente sofre um problema de saúde em consequiência de uma inefetividade não quantitativa do medicamento.

PRM4. O paciente sofre um problema de saúde em conseqüência de uma inefetividade quantitativa do medicamento.

PRM5. O paciente sofre um problema de saúde em conseqüência de uma insegurança não quantitativa do medicamento. 


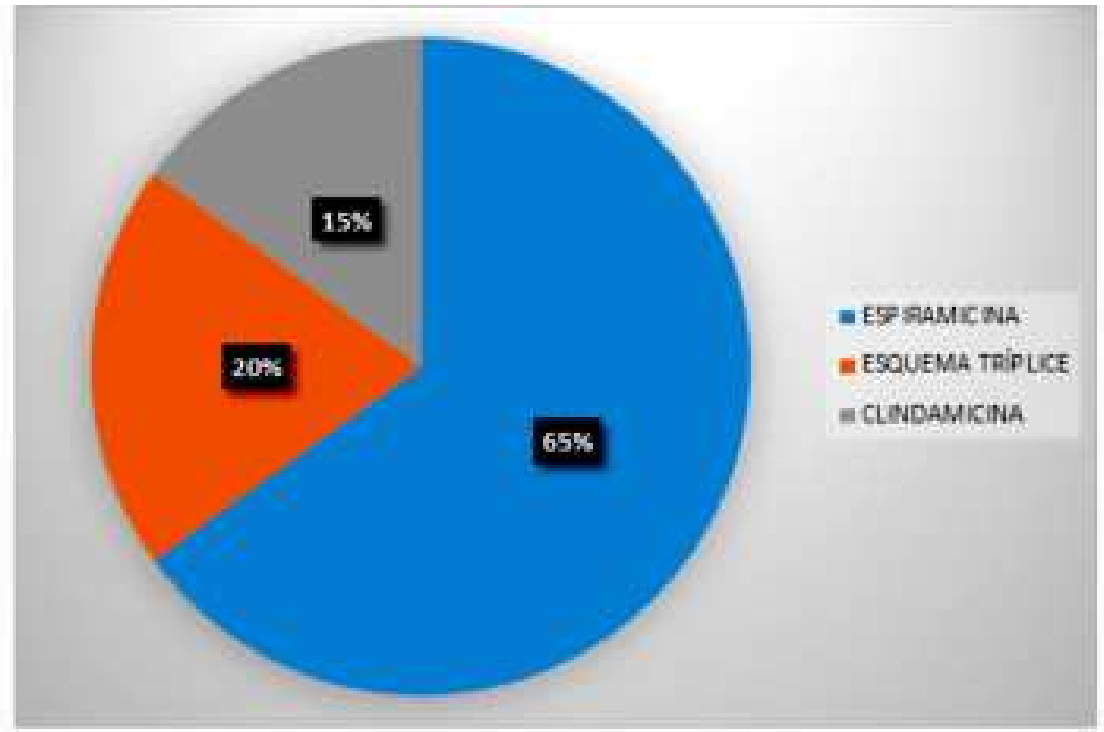

Fig. 2-Medicamentos que causaram efeitos adversos nas gestantes

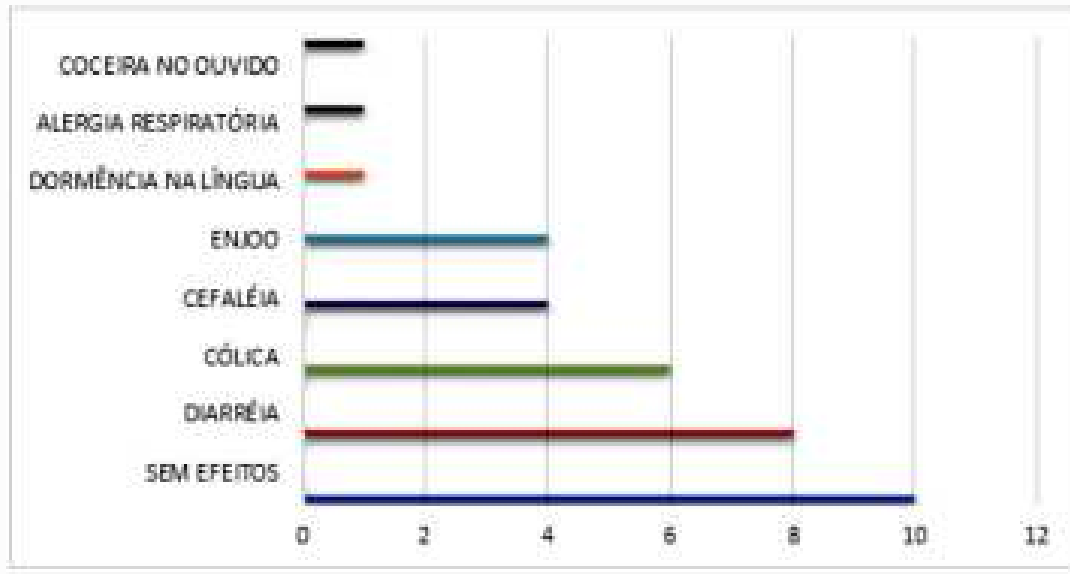

Fig. 3- Efeitos colaterais ou adversos apresentados pelas gestantes durante o tratamento.

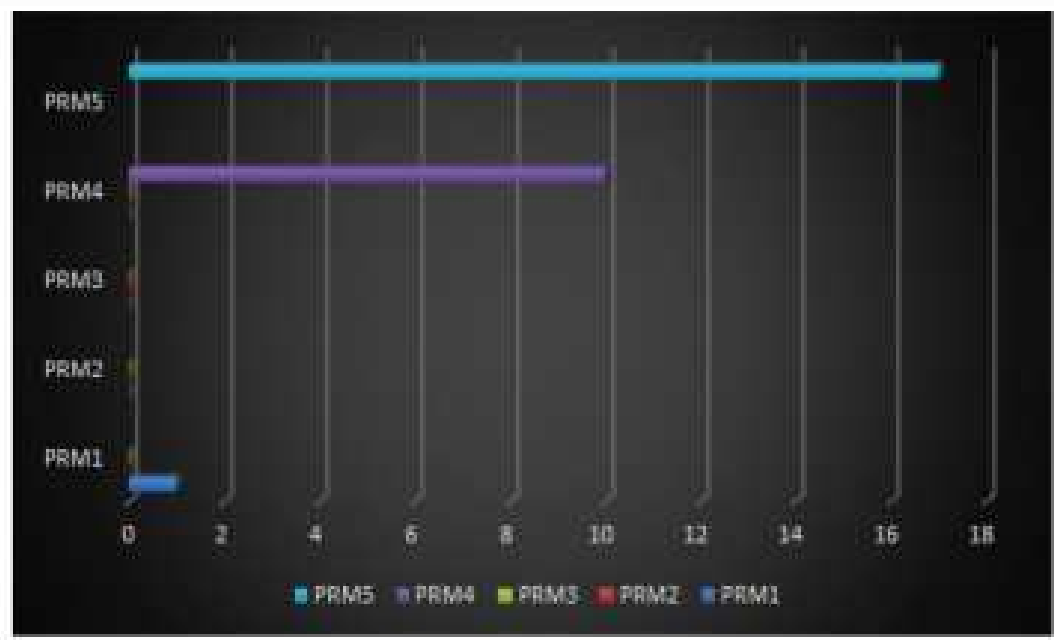

Fig. 4 - PRMs identificados durante o tratamento farmacoterapeutico das qestantes. 
Tabela 2- Medicamentos receitados, e posologia adotada pelas gestantes durante o tratamento para toxoplasmose.

\begin{tabular}{l|l|l|l}
\hline MEDICAMENTO & $\begin{array}{l}\text { QUANTIDADE } \\
\text { COMPRIMIDOS }\end{array}$ & HORARIOS & $\begin{array}{l}\text { NUMERO DE } \\
\text { GESTANTES }\end{array}$ \\
\hline ESPIRAMICINA & 1 & $8 / 8$ HORAS & 10 \\
\hline ESPIRAMICINA & 1 & $\begin{array}{l}12 / 12 \\
\text { HORAS }\end{array}$ & 6 \\
\hline ESPIRAMICINA & 2 & $\begin{array}{l}\text { UMA VEZ } \\
\text { AO DIA }\end{array}$ & 1 \\
\hline CLINDAMICINA & 1 & $\begin{array}{l}\text { UMA VEZ } \\
\text { AO DIA }\end{array}$ & 2 \\
\hline RIFAMICINA & 1 & $8 / 8$ HORAS & 1 \\
\hline RIFAMICINA & 1 & $\begin{array}{l}\text { UMA VEZ } \\
\text { AO DIA }\end{array}$ & 1 \\
\hline $\begin{array}{l}\text { ESQUEMA } \\
\text { TRIPLICE }\end{array}$ & 1 & $\begin{array}{l}\text { UMA VEZ } \\
\text { AO DIA }\end{array}$ & 1 \\
\hline $\begin{array}{l}\text { ESQUEMA } \\
\text { TRIPLICE }\end{array}$ & 1 & $8 / 8$ HORAS & 2 \\
\hline $\begin{array}{l}\text { ESQUEMA } \\
\text { TRIPLICE }\end{array}$ & NĀO SABE & NĀO SABE & 6 \\
\hline
\end{tabular}

PRM6. O paciente sofre um problema de saúde em conseqüência de uma insegurança quantitativa.

No estudo observou-se que o PRM5 ocorreu com maior frequência nas gestantes, em 56,6\%. O PRM5 está relacionado a segurança no uso do medicamento, sendo relativo aos efeitos adversos independente da dosagem do medicamento, uma vez que as mesmas tomavam em média 4 comprimidos por dia, e que os medicamentos utilizados (espiramicina, sulfadiazina de prata, ácido folínico, pirimetamina e clindamicina) podem ocasionar várias interações medicamentosas, levando as gestantes tomarem uma dose inferior a de que necessitam.

Segundo as gestantes entrevistadas a adoção da prática de tomar uma dosagem menor que a necessária ocorreu devido os medicamentos causarem muitos efeitos adversos. O uso incorreto de a medicação prescrita gerar o PRM4, que está relacionado a efetividade do medicamento. 17 (56,6\%) gestantes relataram que o uso de doses foi menor que deveriam e um grande intervalo de tempo. Nesses casos, os medicamentos foram menos efetivos durante o tratamento. O estudo mostra que em 10 (33,3\%) das gestantes não ocorreram efeitos adversos.

Foi observado que uma gestante apresentou o PRM1, que está relacionado a prescrição de acordo com a necessidade do paciente, nesse caso, por um período de 2 meses a gestante não realizou o tratamento devido à falta dos medicamentos na farmácia municipal, ou seja a gestante não fez uso dos medicamentos que necessitava gerando o PRM1.

\section{CONCLUSÃO}

A toxoplasmose congênita é uma doença infecciosa que resulta da transferência transplacentária do Toxoplasma gondii para o concepto, decorrente de infecção primária da mãe durante a gestação ou por reagudização de infecção prévia em mães imunodeprimidas (COSTA, 2009).

No presente estudo observou-se a existência de problemas relacionados aos medicamentos por gestantes com toxoplasmose em Campos dos Goytacazes-RJ, 17 (56,6\%) apresentaram o PRM5, $10(33,3 \%)$ o PRM4 e $1(3,33 \%)$ apresentou o PRM1. 
Sugere-se que a avaliação de PRM's em gestantes com toxoplasmose podem ser úteis para o desenvolvimento de processos que visem reduzir esses riscos, tais como a participação de um profissional farmacêutico clínico na equipe de atendimento as gestantes, resultando em um aumento positivo nos resultados da farmacoterapia e, consequentemente trazendo benefícios para as mesmas.
Os farmacêuticos são profissionais que podem desempenhar um papel relevante nessa monitorização, colaborando juntamente com médicos e pacientes gestantes na garantia de um tratamento seguro e efetivo, fazendo também uma avaliação farmacêutica na prescrição e na forma de como esses medicamentos estão sendo utilizados por elas, essas medidas podem gerar melhores resultados durante o tratamento e diminuir os números de PRMs.

\section{REFERÊNCIAS BIBLIOGRÁFICAS}

AIZESTEIN, M. TOMASSI, M. Problemas relacionados a medicamentos; reações adversas a medicamentos e erros de medicação: a necessidade de uma padronização nas definições e classificações. Revista Cientifica de Farmacêuticas Básica e Aplicada, v. 32, n2, p. 170-173, fev.2011.

ANVISA. Daraprim. Bula do Paciente. Disponível em:<file://F:/fmrVisualizarBula\%20bula\%201.pdf>. Acesso em: 5 out.2016. ANVISA. Azulfin. Bula do paciente. Disponível em: <file://F:/AZULFIN Bula do paciente V01\%20BULA\%202.pdf> . Acesso em: 5 out. 2016.

ANVISA. Periodantil. Bula do paciente. Disponível em: <file://F:/frmVisualizarbula\%203.pdf > . Acesso em: 5 out.2016.

CÂMARA, J. SILVA, M. CASTRO, A. Prevalência de Toxoplasmos em gestantes atendidas em dois centros de referência em uma cidade do Nordeste, Brasil, Revista Brasileira de Ginecologia e Obstetrícia, v. 37 n.2, p. 65-69, jul. 2014.

CORRER, C. J. et al. Riscos de problemas relacionados com medicamentos em pacientes de uma instituição geriátrica, Revista Brasileira de Ciências Farmacêuticas, v. 43 n. 1, p. 55-62, jan/mar. 2007.

COSTA, I. N. Avaliação da eficácia dos tratamentos com associação de Sulfadiazina, Pirimetamida e Ácido folínico, Azitromicina, Infusão de ArtenisiaAnnua L. e Espiramicina na prevenção da transmissão vertical da toxoplasmose em CalomysCallosus. Uberlândia, 2009. 94p. Dissertação (Mestrado) - Universidade Federal de Uberlândia. Instituto de ciências biomédicas, 2009. Disponível em: >https://repositorio.ufu.br/bitstream/123456789/16555/1/aIdessania.pdf > . Acesso em: 22 març.2017.

MARGONATO, F. et al. Toxoplasmose na gestação: diagnóstico, tratamento e importância de protocolo clínico, Revista Brasileira de Saúde Materno Infantil, v. 7 n. 4, p. 381-386, ago. 2007.

MARTINS, C. Apresentação Clínica da Toxoplasmose Congênita.Toxoplasmose na gravidez, v. 18, n.5.2002.

NASCIMENTO, I. et al. Estudo da Prevalência de anticorpos anti-toxoplasmagondii em mulheres grávidas no Estado da Bahia. Revista de Ciências Médicas e Biológicas, v.1, n. 1, 2002.

SÁ, R. JUNIOR, M. NETTO, H. Toxoplasmose Congênita. Jornal Brasileiro de Ginecologia, v. 107 n.8, p. 281-286, ago. 1997. 